\title{
Multichannel switching in a multigap gas switch at atmospheric pressure
}

\author{
A. A. Zherlitsyn® and E. V. Kumpyak \\ Institute of High Current Electronics, 2/3 Akademichesky Avenue, Tomsk, 634055, Russia
}

(Received 30 April 2020; accepted 12 October 2020; published 27 October 2020)

\begin{abstract}
The multichannel switching mode of a controlled multigap switch is studied. The switch is designed for capacitive energy storage, with a charging voltage of up to $100 \mathrm{kV}$ and an energy output time of about $100 \mathrm{~ns}$. The interval between the high-voltage and low-voltage electrodes of this switch is divided into seven series-connected gaps using ball electrodes, and six parallel rows of these ball electrodes allow for multichannel switching. An optical system based on collimators and photodiodes was used to determine the number of channels in the switch gaps. Statistics on the number of channels were collected and the probability of ignition of parallel spark channels was calculated for each of the switch gaps under different operating conditions. We show that both the electrical isolation between the channels and the rate of rise of the triggering pulse significantly affect the number of parallel ignited channels and their distribution over consecutive gaps. At a high triggering voltage pulse rise rate $(\sim 800 \mathrm{kV} / \mu \mathrm{s})$, a larger number of spark channels are ignited in the switch with electrical isolation between the channels and the characteristics of this switch are better. At a low triggering voltage pulse rise rate (less than $250 \mathrm{kV} \mu \mathrm{s}$ ), better characteristics are realized in the switch without electrical isolation between the channels.
\end{abstract}

DOI: 10.1103/PhysRevAccelBeams.23.100402

\section{INTRODUCTION}

Linear transformer drivers (LTDs) are under active development for high-voltage, high-current pulsed-power generators, and may significantly reduce their cost and size [1-5]. LTDs are based on the use of a primary capacitive storage unit with an energy output time of about 100 ns. The capacitive energy storage is divided into sections, each switched by its own gas spark switch. The number of switches can considerably exceed $10^{4}$ in the newly developed petawatt-class LTDs [6-8]. The parameters of the primary energy storage device and LTD depend on the parameters of the switch, and studies of the different types of gas spark switch are therefore given special attention [9-14].

One avenue for the development of LTD generators is the use of atmospheric pressure air as insulation for the LTD stages and the working medium of the switches [15-17]. This requires increasing the length of the surface between the high-voltage and low-voltage electrodes of the switch, and for this, the planar geometry of the switch is used [16-19]. The interelectrode gap of the planar switch is divided into a series of connected small gaps using ball

Published by the American Physical Society under the terms of the Creative Commons Attribution 4.0 International license. Further distribution of this work must maintain attribution to the author(s) and the published article's title, journal citation, and DOI. electrodes, and parallel rows of these ball electrodes allow for multichannel switching. The important parameters of the switch, such as its resistance, inductance, dissipated energy and lifetime, are determined by the number of parallel ignited channels.

The switch and its triggering system should be considered together, as a single coupled system, when solving the multichannel switching problem. Obviously, the higher the amplitude and rise rate of the triggering voltage pulse, the better. However, insulation requirements are increasing and the technical implementation of the triggering system is becoming more complicated, especially for multisection installations. It is necessary to optimize the parameters of the triggering system that provide the multichannel switching mode, and optical diagnostic methods can be used to study multichannel switching and optimize these parameters [10,20,21].

This paper presents the results of a study of the probability of ignition of parallel spark channels in a multigap gas switch at atmospheric pressure under different operating conditions, utilizing an optical diagnostic system. We studied the influence of the rate of rise of the triggering voltage in the range of $100-800 \mathrm{kV} / \mu \mathrm{s}$, the electrical decoupling between the channels and the auxiliary discharge of ionizing radiation on the number of channels. The dissipated energy of parallel spark channels was calculated based on experimental data on the number of channels. The calculation results allow us to reasonably choose the parameters of the triggering pulse and the design of the switch. 


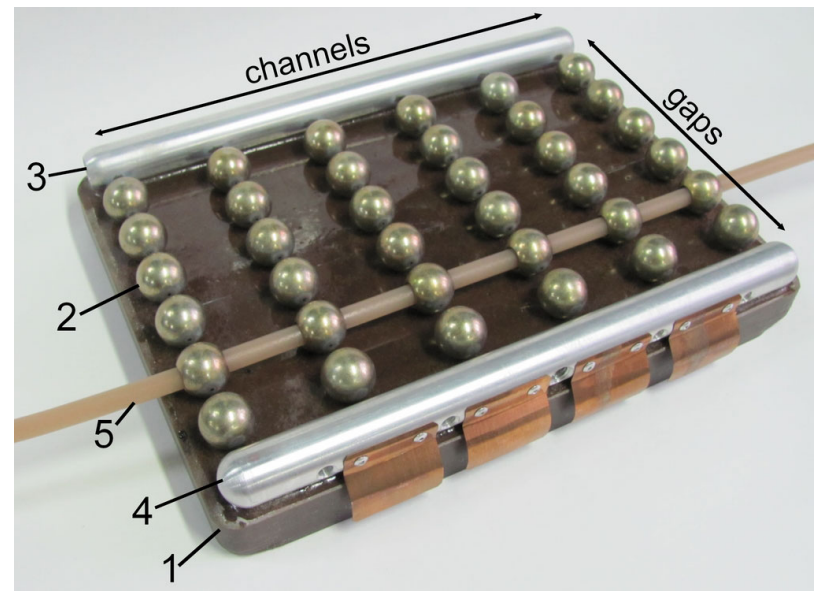

FIG. 1. Design of the switch with isolation between the channels: (1) epoxy slab; (2) intermediate ball electrodes; $(3,4)$ high- and low-voltage end electrodes; (5) triggering wire.

\section{APPARATUS}

\section{A. Switch}

The design of the switch is shown in Fig. 1. The gap between the high-voltage (3) and low-voltage (4) electrodes is divided into seven serial gaps using intermediate electrodes (2). The high-voltage and low-voltage electrodes are cylinders with a diameter of $22 \mathrm{~mm}$, while the intermediate electrodes are balls with the same diameter. The length of a single gap is $6 \mathrm{~mm}$, giving a total gap for the switches of $42 \mathrm{~mm}$. The ball electrodes are divided into six groups, forming six parallel channels. The switch electrodes are assembled on an epoxy slab (1).

The voltage across the gaps is distributed using a resistive voltage divider, in which the resistors are connected in series in one of the channels between the highand low-voltage electrodes. Conducting rubber cords run in parallel to the high- and low-voltage electrodes inside the slab, ensuring that the voltage is distributed over the electrodes in all the other channels. The resistance of the rubber between adjacent channels is $\sim 0.8 \mathrm{M} \Omega$.

A triggering pulse is supplied to each of the channels to the electrodes of the third row, relative to the grounded side. These electrodes contain holes of diameter $9 \mathrm{~mm}$, through which a triggering wire (5) with diameter of $3 \mathrm{~mm}$ passes with polyethylene insulation.

The dimensions of the switch are $298 \times 220 \times 50 \mathrm{~mm}^{3}$.

An equivalent electrical scheme of the switch during charging of the capacitive storage device and an electrical scheme for a single switch channel at triggering are shown in Fig. 2. During charging, the voltage is distributed uniformly over the switch electrodes. At a charging voltage of $100 \mathrm{kV}$, the potential difference between the electrodes $\left(U_{\text {gap }}=U_{0} / 7\right)$ is less than $14.5 \mathrm{kV}$. The self-breakdown voltage of the gaps in air at atmospheric pressure is $18-19 \mathrm{kV}$.

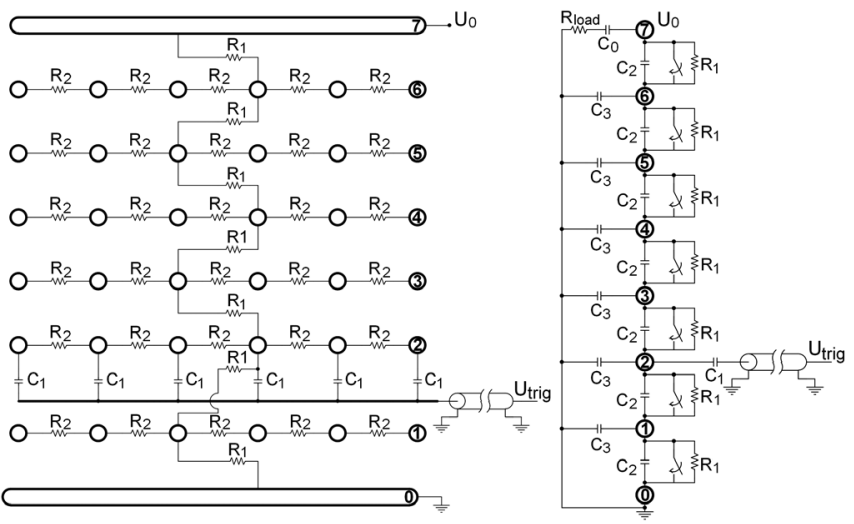

(a)

(b)

FIG. 2. (a) Equivalent electrical scheme for the switch during charging of the capacitive storage device; (b) scheme for one channel at triggering. $R_{1}=68 \mathrm{M} \Omega$-divider resistors; $R_{2}$ $0.8 \mathrm{M} \Omega$ - cord piece resistance; $C_{1} \sim 2.3 \mathrm{pF}$-capacitance between triggering wire and ball electrode; $C_{2} \sim 1 \mathrm{pF}$-capacitance between electrodes; $C_{3} \sim 1.8 \mathrm{pF}$-capacitance of ball electrodes to Earth.

If there is a breakdown in the gap of one of the channels, then the voltage at the electrodes of the adjacent channels will change with an $R C$ time constant $\tau=R_{2} C_{3} \sim 1 \mu \mathrm{s}$. Since $\tau$ is much greater than the breakdown time for the gaps of one channel, the channels can be considered as electrically isolated and independent of each other, as shown in the electrical scheme in Fig. 2(b).

When a triggering voltage $\left(U_{\text {trig }}\right)$ with polarity opposite to the main voltage is applied, the uniform voltage distribution over the electrodes is disturbed and breakdown is initiated. The calculated voltage distribution over the electrodes of one channel as breakdown progresses is shown in Fig. 3. In this example, the switch voltage is $100 \mathrm{kV}$, and the amplitude of the triggering pulse is $80 \mathrm{kV}$. The fraction of the triggering voltage applied to triggering electrode 2 is determined by the following expression:

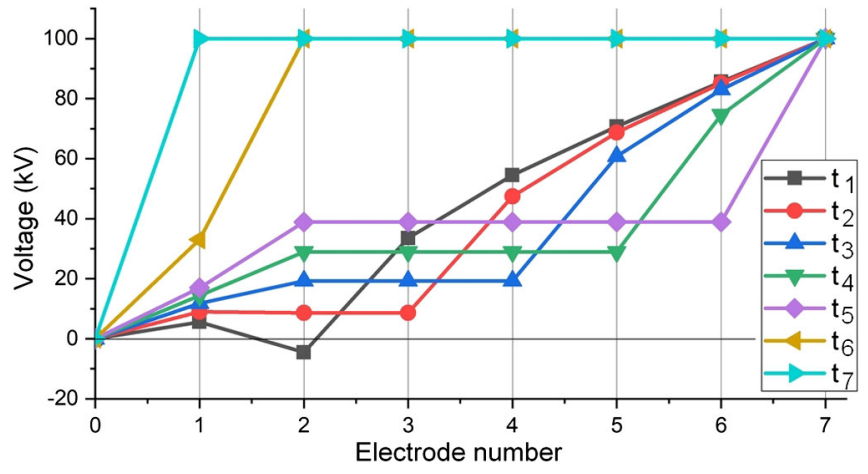

FIG. 3. Voltage distribution over the switch electrodes after the triggering pulse is applied $\left(t_{1}\right)$ and after the breakdown of the gaps between electrodes 2-3 $\left(t_{2}\right), 3-4\left(t_{3}\right), 4-5\left(t_{4}\right), 5-6\left(t_{5}\right)$, 6-7 $\left(t_{6}\right)$ and 2-1 $\left(t_{7}\right)$. 


$$
U_{\text {trig, }, 2} \sim U_{\text {trig }} \frac{C_{1}}{C_{1}+C_{3}+2 \cdot C_{2}} \sim 0.41 \cdot U_{\text {trig }} .
$$

The potential from a triggering pulse on adjacent electrodes is described by the following expressions [22]:

$$
\begin{aligned}
& U_{\text {trig }, 3}=U_{\text {trig }, 2} \frac{\operatorname{sh}(5 \cdot a)}{\operatorname{sh}(6 \cdot a)} \approx 0.117 \cdot U_{\text {trig }} \\
& U_{\text {trig }, 4}=U_{\text {trig }, 2} \frac{\operatorname{sh}(4 \cdot a)}{\operatorname{sh}(6 \cdot a)} \approx 0.033 \cdot U_{\text {trig }} \\
& U_{\text {trig }, 5}=U_{\text {trig, }, 2} \frac{\operatorname{sh}(3 \cdot a)}{\operatorname{sh}(6 \cdot a)} \approx 0.009 \cdot U_{\text {trig }} \\
& U_{\text {trig }, 6}=U_{\text {trig }, 2} \frac{\operatorname{sh}(2 \cdot a)}{\operatorname{sh}(6 \cdot a)} \approx 0.003 \cdot U_{\text {trig }} \\
& U_{\text {trig }, 1}=U_{\text {trig, }, 2} \frac{\operatorname{sh}(1 \cdot a)}{\operatorname{sh}(2 \cdot a)} \approx 0.108 \cdot U_{\text {trig }},
\end{aligned}
$$

where $a=\ln \left[1+\frac{C_{1}}{2 \cdot C_{2}} \cdot\left(1+\sqrt{\frac{4 \cdot C_{2}}{C_{1}}+1}\right)\right]$.

The voltage in the gaps on different sides of triggering electrode 2 is not the same. The voltage in the gaps between electrodes 2 and 3 and electrodes 1 and 2, taking into account the initial distribution of the charging voltage $\left(U_{0}\right)$, is the following:

$$
\begin{aligned}
U_{3}-U_{2} & =\frac{U_{0}}{7}+\left(U_{\text {trig }, 3}-U_{\text {trig }, 2}\right) \\
& \approx U_{0} / 7-0.293 \cdot U_{\text {trig }}, \\
U_{2}-U_{1} & =\frac{U_{0}}{7}+\left(U_{\text {trig }, 2}-U_{\text {trig }, 1}\right) \\
& \approx U_{0} / 7+0.302 \cdot U_{\text {trig }} .
\end{aligned}
$$

A higher voltage is generated in the gap between electrodes 2 and 3 according to expressions (7) and (8) and taking into account the different polarity of the charging voltage $\left(U_{0}\right)$ and the triggering voltage $\left(U_{\text {trig }}\right)$ (time $t_{1}$ ). The voltage in this gap increases by a factor of more than 2.5, which leads to the breakdown of this gap. After breakdown of the gap between electrodes 2 and 3, a triggering voltage is applied to electrode 3 (time $t_{2}$ ), and breakdown of the gap between electrodes 3 and 4 occurs, and so on (time $t_{3}-t_{5}$ ). After breakdown of the gaps between electrodes 2 and 7, the capacitive storage voltage is applied to the gaps between electrodes 0 and 1 and electrodes 1 and 2 (time $t_{6}$ ). Breakdown of the last gaps can occur when the voltage is more than 4 times higher than the initial value. It can be seen that as the gaps are broken down, the possible overvoltage at the unbroken gaps increases.
The number of parallel spark channels in the gaps cannot exceed the number of channels in the gap, which breaks first (the gap between electrodes 2 and 3), since the electrodes of one row are electrically isolated $\left(R_{2}\right.$ is very high) and the discharge current cannot be redistributed between them. The maximum possible number of parallel spark channels in the gap is equal to the number of ball electrodes in the row, i.e., six. The actual number of channels is determined by the rate of increase in the triggering voltage pulse.

However, if the electrodes of one row are electrically connected $\left(R_{2} \approx 0\right)$, then the discharge current can be redistributed between the electrodes of the same row, and a different number of channels can be ignited in the gaps. We expect an increase in the number of parallel channels with the successive breakdown of gaps due to an increase in overvoltage. To verify this, changes were made to the design of the multichannel multigap switch.

The design of the modified switch is shown in Fig. 4. We removed the electrical isolation between the channels in this version. Ball electrodes in one row are connected by stainless steel rods with a diameter of $6 \mathrm{~mm}$ [thus $R_{2}=0$ in Fig. 2(a)]. The design of the switch allowed us to install serrated stainless steel blades on the rods, as shown in Fig. 4. These blades are intended to give ignition of the corona discharge when the capacitive storage device is charged. The thickness of the blades was $0.2 \mathrm{~mm}$, and their length was selected to give corona discharge ignition at the blade at low voltage and to ensure that triggering breakdown did not occur from the blade but from a ball electrode. The corona discharge current was $\sim 1 \mu \mathrm{A}$ at a switch voltage of about $70 \mathrm{kV}$, increasing to $\sim 100 \mu \mathrm{A}$ as the voltage increased to $100 \mathrm{kV}$.

The triggering system remained the same, and the triggering pulse was supplied through separation capacitances to each electrode in the third row, relative

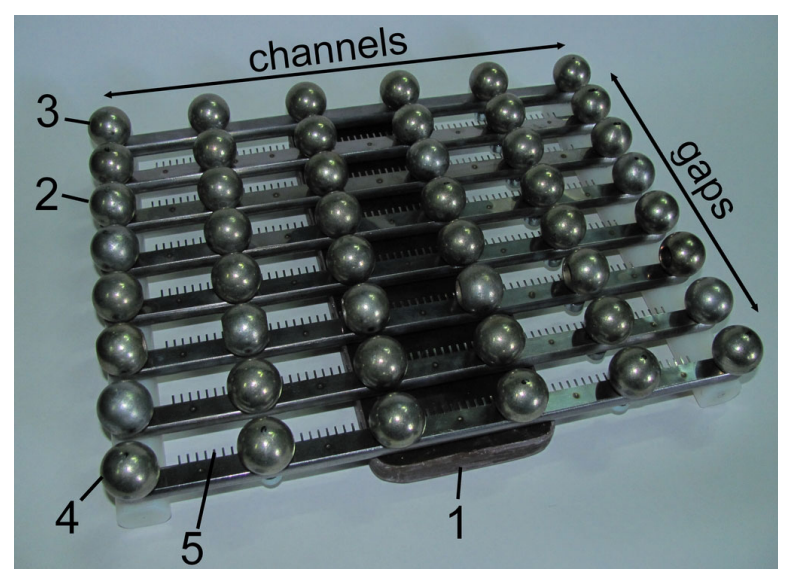

FIG. 4. Design of the switch without isolation between the channels: (1) resistive voltage divider inside epoxy slab; (2) intermediate ball electrodes; $(3,4)$ high- and low-voltage end electrodes; (5) corona discharge blades. 


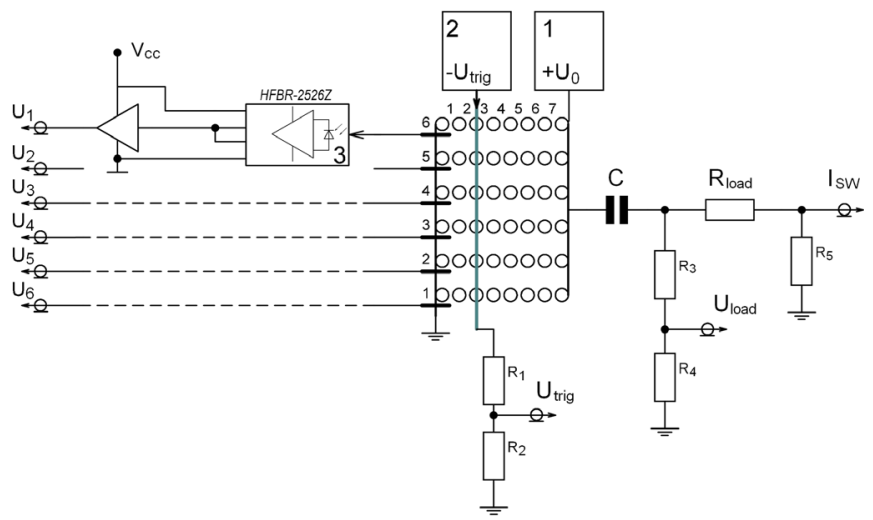

FIG. 5. Scheme of the test bed for switch testing: (1) HV power supply; (2) triggering generator; (3) photodiode and preamplifier circuit.

to the grounded side. The dimensions of the switch were unchanged.

\section{B. Switch-testing apparatus}

A scheme for a test bed for the switch tests is shown in Fig. 5. A capacitive energy storage device $C=20 \mathrm{nF}$ (two series-connected capacitors from General Atomics GA35426, $100 \mathrm{kV}, 40 \mathrm{nF}$ ) is charged with a positive polarity voltage of up to $100 \mathrm{kV}$ from an high voltage (HV) power supply (1). The switch is triggered by a negative polarity voltage pulse from a triggering generator (2). Three triggering pulse generators with voltage rise rates of 100 , 250 and $800 \mathrm{kV} / \mu$ s were used (see Fig. 6). After switch triggering, the capacitive storage is discharged via a load $R_{\text {load }}=3.8 \Omega$.

The triggering voltage $U_{\text {trig }}(t)$ is monitored using the resistive divider $R_{1}-R_{2}$; the load voltage $U_{\text {load }}(t)$ is monitored through the resistive divider $R_{3}-R_{4}$; and the switch current $I_{\mathrm{SW}}(t)$ is monitored through the resistive shunt $R_{5}$. Figure 7 shows an example of current waveforms when using the switch without electrical isolation between channels for three cases with different triggering voltage rise rates. A decrease in the amplitude and an increase in the

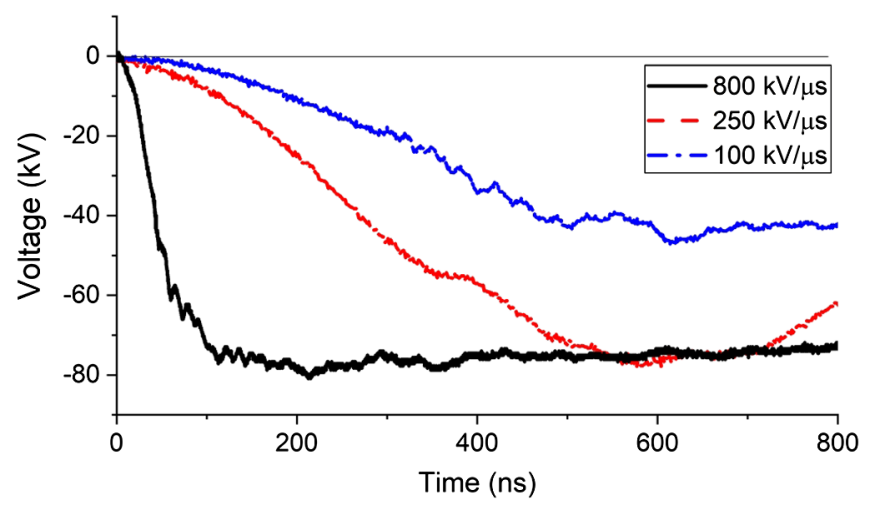

FIG. 6. Triggering pulse waveforms.

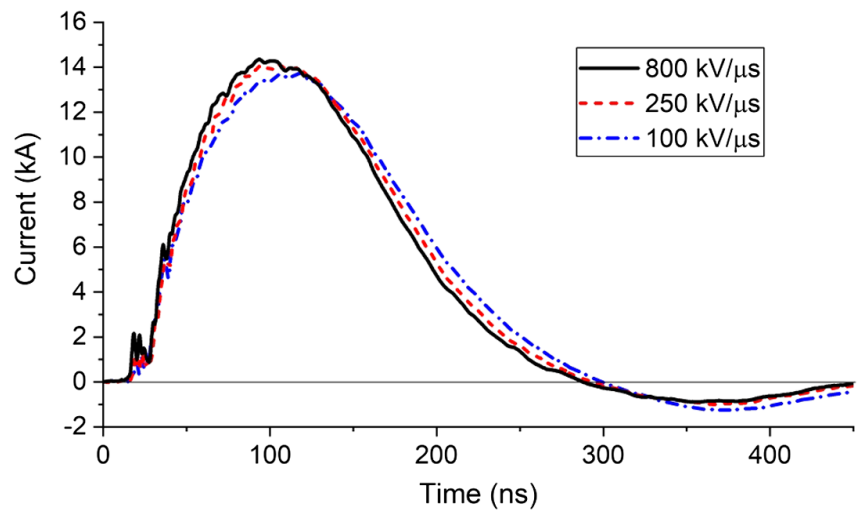

FIG. 7. Switch current waveforms.

current pulse front with a decrease in the triggering pulse rise rate is associated with a decrease in the number of parallel channels.

An optical system is used to determine the number of channels in the switch gaps. The system consists of six collimators with an extended hole and the light is fed through a fiber to a photodiode with a preamplifier circuit (HFBR-25X6Z, Avago Technologies). Six signals $U_{1}-U_{6}$ from each photodiode are fed into an amplifier and then recorded using Tektronix TDS 3054B digital oscilloscopes with a bandwidth of $500 \mathrm{MHz}$ (see Fig. 5). The time resolution of the photodiodes and amplification circuit is a few nanoseconds.

The arrangement of the collimators in the switch is shown in Fig. 8. Six collimators were arranged in a row in one of seven positions. Each position corresponds to one of the seven gaps of the switch. The dimensions and arrangement of the collimators are adjusted to exclude light from neighboring gaps, i.e., each collimator only registers light from the gap over which it is placed.

The research method is as follows. Six collimators were placed above gap 1 (see Fig. 8, side view). A series of

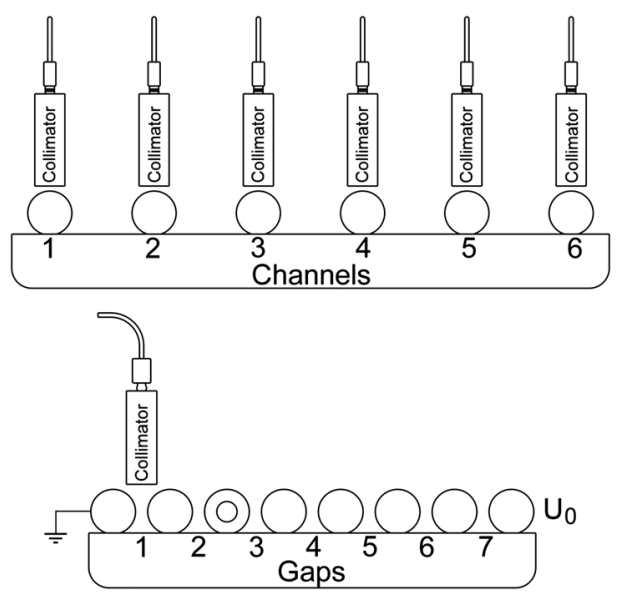

FIG. 8. Front and side views of the switch and arrangement of the collimators. 
$N_{0}=50$ successive pulses was performed. For each pulse, the signals from the photodiodes were recorded, which made it possible to determine how many and which channels were ignited in gap 1 . The number of channel ignition cases $N$ and the probability of ignition $\left(\frac{N}{N_{0}}\right) \cdot 100 \%$ were calculated for each of the six channels in gap 1 . In this calculation, only cases of channel ignition during the front of the discharge current pulse were taken into account. Six collimators were then moved to position 2 above gap 2 . A series of 50 successive pulses was performed again. The number of channel ignition cases and the probability of ignition were calculated for each of the six channels in gap 2. Six collimators were then moved to position 3 above gap 3 and so on. Thus, we sequentially placed the collimators in seven positions and performed 50 pulses for each of them. The full series for calculating the probability of ignition of each of the six channels in each of the seven gaps was 350 pulses under fixed operating conditions.

In addition, the statistics for the delay time between the triggering pulse and the breakdown of the switch were collected using a series of 350 pulses. The delay time was determined as the time between the $10 \%$ levels of the amplitude of the triggering pulse and the load voltage. The statistical delay time was characterized by the average delay time and the standard deviation (jitter of the switch).

Examples of signals from the sensors are given in Fig. 9. In the first example, all six channels are ignited in a gap with a spread of $10 \mathrm{~ns}$, while in the second, five of the six
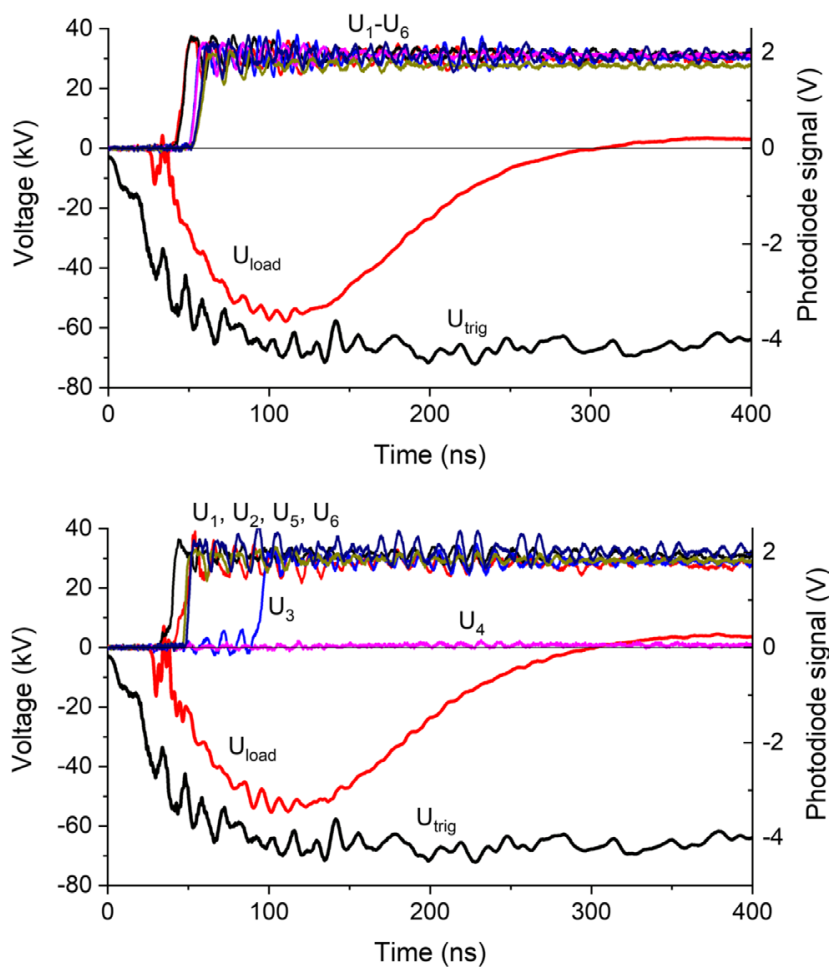

FIG. 9. Triggering pulse waveform $U_{\text {trig }}$, load voltage waveform $U_{\text {load }}$ and signals from six photodiodes $U_{1}-U_{6}$. channels are ignited, and the third channel is ignited with a delay of $50 \mathrm{~ns}$ relative to the remaining channels.

\section{RESULTS}

Figure 10 shows the probabilities of ignition of channels in each of the seven gaps of the switch with electrical isolation between the channels. The results show that the number of channels in all the gaps is approximately the same in the switch with electrical isolation between the channels, and that this is determined by the number of channels initiated by the triggering pulse in gap 3, which breaks first. A rise rate of the triggering pulse of $\sim 800 \mathrm{kV} / \mu$ s provides the ignition of four parallel channels in all gaps with a probability of at least $85 \%$. The number of channels decreases with a decrease in the rise rate of the triggering pulse, and the probability of ignition of four parallel channels in the gaps does not exceed $32 \%$ with a threefold decrease in the rise rate of the triggering pulse.

The situation changes when there is no electrical isolation between the channels. Figure 11 shows the
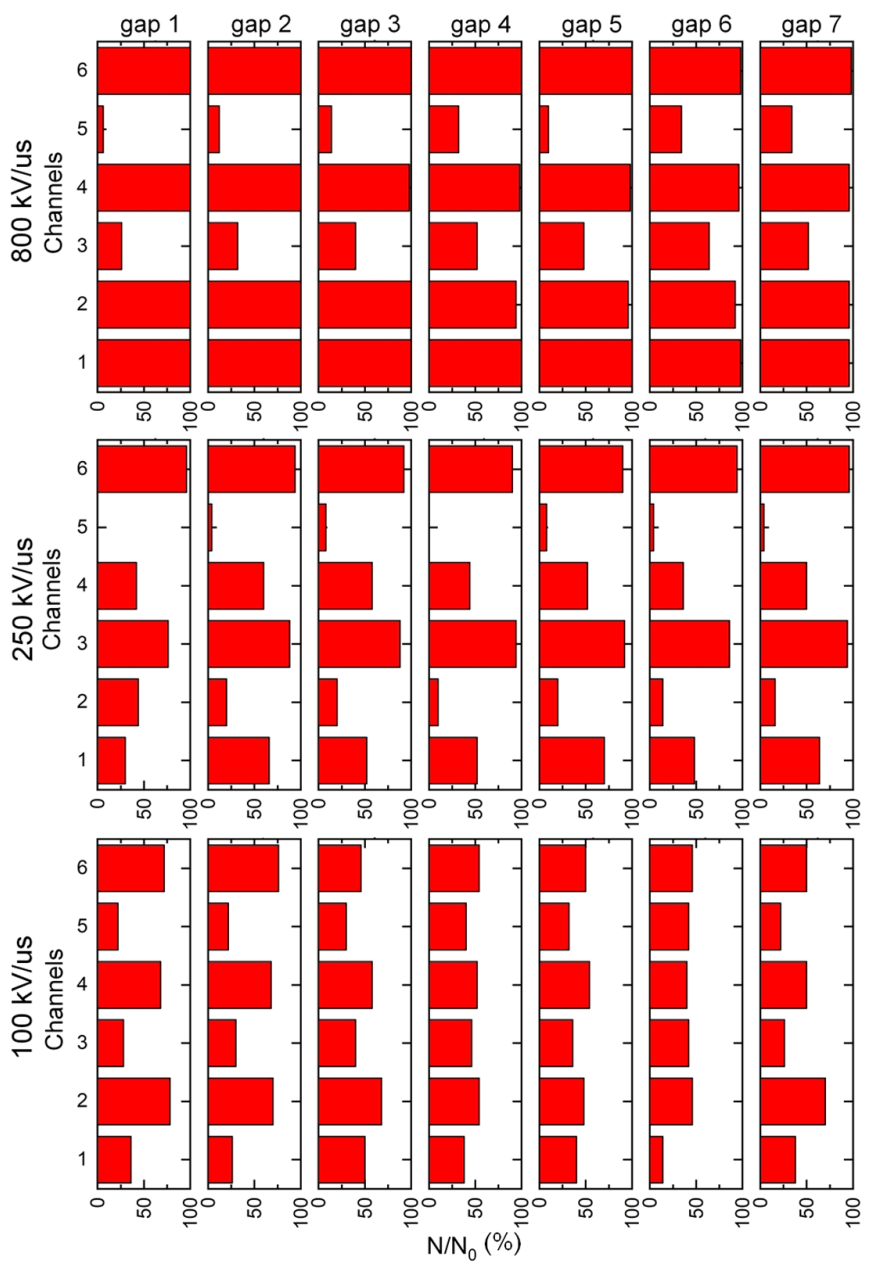

FIG. 10. Probability of ignition of spark channels in the gaps of the switch with electrical isolation between the channels, for rise rates of the triggering pulse of 800,250 and $100 \mathrm{kV} / \mu \mathrm{s}$. 


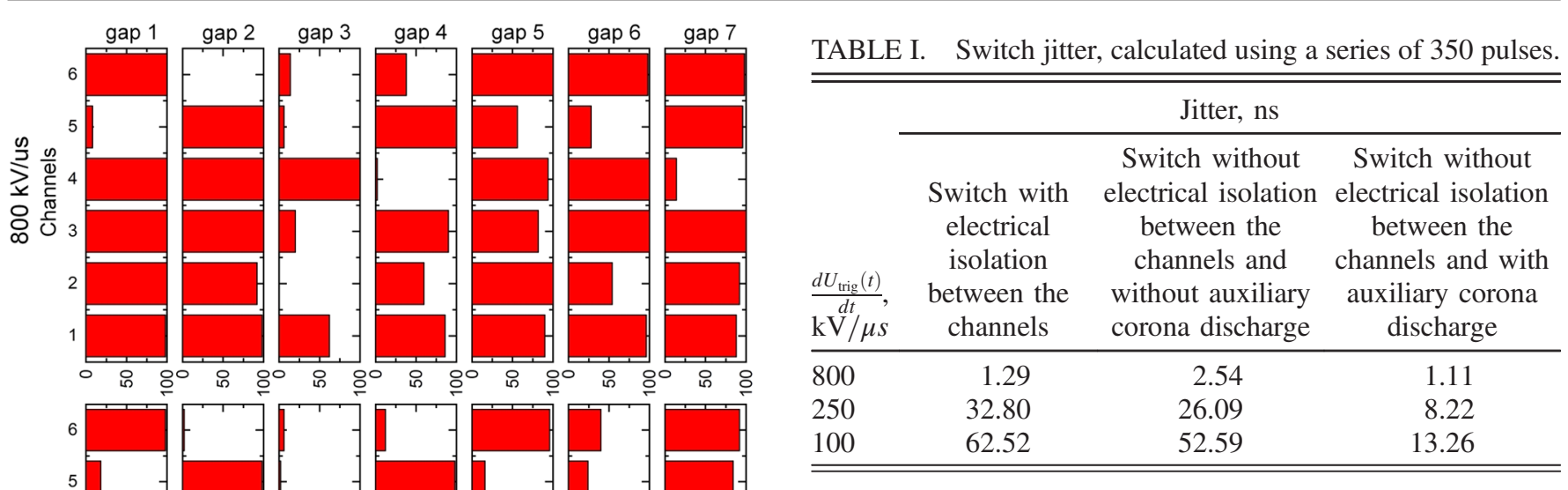

discharge is observed to be significant when trigger pulses with a low rise rate are used. Corona discharge reduces jitter by a factor of 3-4 at a trigger pulse rise rate of below $250 \mathrm{kV} / \mu \mathrm{s}$. For a high rise rate of the triggering pulse $(\sim 800 \mathrm{kV} / \mu \mathrm{s})$, the effect of the corona discharge is less pronounced.

\section{DISCUSSION}

Experimental data on the number of channels make it possible to compare the level of energy loss in the switch in the presence and absence of electrical isolation between the channels. Dissipated energy in the spark channels of a seven-gap switch can be written as

$$
E_{\mathrm{SW}}(t)=\sum_{i=1}^{7} E_{i}(t),
$$

FIG. 11. Probability of ignition of spark channels in the gaps of the switch without electrical isolation between the channels, for rise rates of the triggering pulse of 800,250 and $100 \mathrm{kV} / \mu \mathrm{s}$.

probabilities of ignition of the channels in each of the seven gaps of the switch without electrical isolation between the channels. Only one channel is mainly ignited in the triggering gap 3 . The number of parallel channels increases with the breakdown of subsequent gaps, due to an increase in overvoltage. The largest number of channels is realized in the gaps breaking through last: the gap closest to the capacitive storage (gap 7) and the two gaps at the side of the grounded electrode (gaps 1 and 2). The number of channels decreases with a decrease in the rise rate of the triggering voltage, as expected; however, five channels are ignited in gaps 1 and 2, and four channels are ignited in gap 7, with a probability of more than $75 \%$ even at a rise rate of $100 \mathrm{kV} / \mu \mathrm{s}$.

The additional blade electrodes of the auxiliary corona discharge did not affect the number of channels ignited, although the auxiliary discharge affected the jitter of the switch. Table I shows data on the jitter of the switch for various configurations, depending on the rise rate of the triggering pulse. The stabilizing effect of the corona where $E_{i}(t)$ is the dissipated energy of the system of parallel spark channels in gap $i$. In the calculations, we assumed a uniform distribution of current between parallel channels of the same gap, i.e., the current through a single channel in the gap is

$$
I_{i}(t)=I_{\mathrm{SW}}(t) / N_{i},
$$

where $I_{\mathrm{SW}}(t)$ is the switch current and $N_{i}$ is the number of spark channels in gap $i$.

With a 1-10 kA spark channel current and submicrosecond pulse duration, satisfactory agreement with the experiment is provided by the Braginskii model (hydrodynamic expansion of a spark channel in an approximation of constant conductivity) [23]. The model gives an expression for the radius of the expanding channel with current $I_{i}(t)$ :

$r_{i}(t)=r(0)+\left(\frac{4}{\rho_{0} \pi^{2} \xi \sigma}\right)^{1 / 6}\left(\int_{0}^{t}\left|I_{i}(t)\right|^{2 / 3} d t\right)^{1 / 2}$,

where $r(0) \sim 0.05 \mathrm{~mm}$ and is the initial channel radius, $\rho_{0}$ is the gas density at which the discharge occurs, 
TABLE II. Number of parallel channels with a probability of ignition of more than $90 \%$ and the energy dissipated in the switch at different rise rates of the triggering pulse.

\begin{tabular}{|c|c|c|c|c|c|c|c|c|}
\hline$\frac{\overline{d U_{\text {trig }}(t)}}{d t}$, & & & & & & & & \\
\hline $\mathrm{kV} / \mu s$ & $N_{1}$ & $N_{2}$ & $N_{3}$ & $N_{4}$ & $N_{5}$ & $N_{6}$ & $N_{7}$ & $E_{\mathrm{SW}}, \mathrm{J}$ \\
\hline
\end{tabular}

Switch with electrical isolation between the channels

$\begin{array}{lllllllll}800 & 4 & 4 & 4 & 4 & 4 & 3 & 3 & 13.34\end{array}$

$\begin{array}{lllllllll}250 & 1 & 1 & 1 & 1 & 1 & 1 & 2 & 21.76\end{array}$

$\begin{array}{lllllllll}100 & 1 & 1 & 1 & 1 & 1 & 1 & 1 & 22.54\end{array}$

Switch without electrical isolation between the channels

\begin{tabular}{lllllllll}
800 & 5 & 5 & 1 & 2 & 3 & 4 & 4 & 14.75 \\
250 & 5 & 4 & 1 & 1 & 1 & 2 & 2 & 18.01 \\
100 & 5 & 4 & 1 & 1 & 2 & 1 & 2 & 18.60 \\
\hline
\end{tabular}

$\sigma$ is the specific electrical conductivity and $\xi$ is a dimensionless constant. For air under normal conditions, $\rho_{0}=1.2 \times 10^{-3} \mathrm{~g} / \mathrm{cm}^{3}, \quad \xi \approx 4.5$ and $\sigma=300(\Omega \cdot \mathrm{cm})^{-1}$ [24]. Using (11) and experimental current waveforms, we can calculate the resistance and dissipated energy of a system of $N_{i}$ parallel spark channels in each of the seven gaps.

The resistance of parallel spark channels in a single gap is

$$
R_{i}(t)=\frac{1}{N_{i}} \cdot \frac{l}{\pi r_{i}(t)^{2} \sigma}
$$

The energy dissipated in a single gap is

$$
E_{i}(t)=\int_{0}^{t} I_{\mathrm{SW}}(t)^{2} R_{i}(t) \cdot d t
$$

Table II shows the data on the number of parallel channels in the gaps of the switch. The number of channels ignited with a probability of more than $90 \%$ is taken as the number of spark channels. Table II also shows the energy dissipated in the switch, calculated using Eqs. (9)-(13). At a triggering pulse rise rate of $\sim 800 \mathrm{kV} / \mu \mathrm{s}$, the dissipated energy is $\sim 13.3 \mathrm{~J}$ for the switch with electrical isolation between channels and $\sim 14.7 \mathrm{~J}$ for the switch without electrical isolation between channels. With a decrease in the rise rate of the triggering pulse from 800 to $100 \mathrm{kV} / \mu \mathrm{s}$, the loss of switching energy increases by a factor of 1.7 for the switch with electrical isolation between channels, and less than 1.3 for the switch without electrical isolation. The energy dissipated in the switch without electrical isolation between channels becomes less than in the switch with electrical isolation at a low triggering pulse rise rate $(100-250 \mathrm{kV} / \mu \mathrm{s})$.

\section{SUMMARY}

The number of channels and their distribution over consecutive gaps depends on the electrical isolation between the channels. If there is electrical isolation, the number of channels in all the gaps is approximately the same, and is determined by the number of channels initiated in the triggering gap. A rise rate for the triggering pulse of about $800 \mathrm{kV} / \mu \mathrm{s}$ provides the ignition of four parallel channels in all gaps with a probability of at least $85 \%$. The number of channels decreases with a decrease in the rise rate of the triggering pulse. Hence, the probability of ignition of four parallel channels in the gaps does not exceed $32 \%$ with a threefold decrease in the rise rate.

If there is no electrical isolation, only one channel is mainly ignited in the triggering gap, although the number of parallel channels increases with the successive breakdown of gaps. The largest number of channels is realized in the gaps breaking through last. Four or five channels are ignited in these gaps with a probability of more than $75 \%$, even for a rise rate of the triggering pulse of $100 \mathrm{kV} / \mu \mathrm{s}$. The energy dissipated in this switch is less than in the switch with electrical isolation between the channels at a triggering pulse rise rate of $100-250 \mathrm{kV} / \mu \mathrm{s}$. Thus, the switch without isolation between the channels has an advantage when a trigger pulse with a low rise rate is used.

\section{ACKNOWLEDGMENTS}

The reported study was funded by RFBR, Project No. 18-08-00159.

[1] A. N. Bastrikov, V. A. Vizir, S. N. Volkov, V. G. Durakov, A. M. Efremov, V. B. Zorin, A. A. Kim, B. M. Kovalchuk, E. V. Kumpjak, S. V. Loginov et al., Primary energy storages based on linear transformer stages, Laser Part. Beams 21, 295 (2003).

[2] A. A. Kim, M. G. Mazarakis, V. A. Sinebryukhov, B. M. Kovalchuk, V. A. Visir, S. N. Volkov, F. Bayol, A. N. Bastrikov, V. G. Durakov, S. V. Frolov et al., Development and tests of fast 1-MA linear transformer driver stages, Phys. Rev. ST Accel. Beams 12, 050402 (2009).

[3] W. A. Stygar, W. E. Fowler, K. R. LeChien, F. W. Long, M. G. Mazarakis, G. R. McKee, J. L. McKenney, J. L. Porter, M. E. Savage, B. S. Stoltzfus et al., Shaping the output pulse of a linear-transformer-driver module, Phys. Rev. ST Accel. Beams 12, 030402 (2009).

[4] R. D. McBride, W. A. Stygar, M. E. Cuneo, D. B. Sinars, M. G. Mazarakis, J. J. Leckbee, M. E. Savage, B. T. Hutsel, J. D. Douglass, M. L. Kiefer et al., A primer on pulsed power and linear transformer drivers for high energy density physics applications, IEEE Trans. Plasma Sci. 46, 3928 (2018).

[5] M. G. Mazarakis, W. E. Fowler, K. L. LeChien, F. W. Long, M. K. Matzen, D. H. McDaniel, R. G. McKee, C. L. Olson, J. L. Porter, S. T. Rogowski et al., Highcurrent linear transformer driver development at Sandia National Laboratories, IEEE Trans. Plasma Sci. 38, 704 (2010).

[6] W. A. Stygar, M. E. Cuneo, D. I. Headley, H. C. Ives, R. J. Leeper, M. G. Mazaratis, C. L. Olson, J. L. Porter, 
T. C. Wagoner, and J.R. Woodworth, Architecture of petawatt-class z-pinch accelerators, Phys. Rev. ST Accel. Beams 10, 030401 (2007).

[7] W. A. Stygar, T. J. Awe, J. E. Bailey, N. L. Bennett, E. W. Breden, E. M. Campbell, R. E. Clark, R. A. Cooper, M. E. Cuneo, J. B. Ennis et al., Conceptual designs of two petawatt-class pulsed-power accelerators for highenergy-density-physics experiments, Phys. Rev. ST Accel. Beams 18, 110401 (2015).

[8] L. Chen, W. Zou, L. Zhou, M. Wang, Yu Liu, L. Liu, M. Deng, D. Liu, J. Zhu, K. Lian et al., Development of a fusion-oriented pulsed power module, Phys. Rev. ST Accel. Beams 22, 030401 (2019).

[9] A. A. Kim, B. M. Kovalchuk, V. V. Kremnev, E. V. Kumpyak, A. A. Novikov, B. Etlicher, L. Frescaline, J. F. Leon, B. Roques, F. Lassalle, R. Lample, G. Avrillaud, and F. Kovacs, Multigap, multichannel spark switches, in Proceedings of the 11th IEEE International Pulsed Power Conference, Baltimore, Maryland USA, 1997, edited by G. Cooperstein and I. Vitkovitsky (IEEE, Piscataway, NJ, 1997), pp. 862-867.

[10] J. R. Woodworth, J. A. Alexander, F. R. Gruner, W. A. Stygar, M. J. Harden, J. R. Blickem, G. J. Dension, F. E. White, L. M. Lucero, H. D. Anderson et al., Lowinductance gas switches for linear transformer drivers, Phys. Rev. ST Accel. Beams 12, 060401 (2009).

[11] J. R. Woodworth, W. A. Stygar, L. F. Bennett, M. G. Mazarakis, H. D. Anderson, M. J. Harden, J. R. Blickem, F. R. Gruner, and R. White, New low inductance gas switches for linear transformer drivers, Phys. Rev. ST Accel. Beams 13, 080401 (2010).

[12] X. Liu, F. Sun, T. Liang, X. Jiang, Q. Zhang, and A. Qiu, Experimental study on multigap multichannel gas spark closing switch for LTD, IEEE Trans. Plasma Sci. 37, 1318 (2009).

[13] X. Liu, F. Sun, T. Liang, X. Jiang, Q. Zhang, and A. Qiu, Study on firing conditions of multigap gas switch for fast linear transformer driver, IEEE Trans. Plasma Sci. 38, 1670 (2010).

[14] J. D. Douglass, B. T. Hutsel, J. J. Leckbee, T. D. Mulville, B. S. Stoltzfus, M. L. Wisher, M. E. Savage, W. A. Stygar,
E. W. Breden, J. D. Calhoun et al., 100 GW linear transformer driver cavity: Design, simulations, and performance, Phys. Rev. Accel. Beams 21, 120401 (2018).

[15] B. M. Kovalchuk, A. V. Kharlov, S. N. Volkov, A. A. Zherlitsyn, V. B. Zorin, G. V. Smorudov, and V. N. Kiselev, Electron-beam accelerator for pumping of a $\mathrm{Xe}_{2}$ lamp, Laser Part. Beams 30, 23 (2012).

[16] B. M. Kovalchuk, A. V. Kharlov, E. V. Kumpyak, and A. A. Zherlitsyn, Pulsed generators based on air-insulated linear-transformer-driver stages, Phys. Rev. ST Accel. Beams 16, 050401 (2013).

[17] A. A. Zherlitryn, B. M. Kovalchuk, and G. V. Smorudov, Capacitor units with air insulation for linear transformers, Instrum. Exp. Tech. 52, 802 (2009).

[18] B. M. Kovalchuk, A. V. Kharlov, V. B. Zorin, and A. A. Zherlitsyn, A compact submicrosecond, high current generator, Rev. Sci. Instrum. 80, 083504 (2009).

[19] B. M. Kovalchuk, A. V. Kharlov, E. V. Kumpyak, G. V. Smorudov, and A. A. Zherlitsyn, Capacitor blocks for linear transformer driver stages, Rev. Sci. Instrum. 85, 013501 (2014).

[20] K. Hahn, J. R. Woodworth, W. T Clark, J. R. Blickem, R. Starbird, M. J. Hardin, and Y. Maron, Spectroscopic studies of gas switches for linear transcharacteristics of the newly designed multigap gas former drivers, in Proceedings of the 16th IEEE International Pulsed Power Conference, Albuquerque, NM (IEEE, Piscataway, NJ, 2007), pp. 125-128.

[21] S. Liang, L. Yang, S. Tieping, C. Peitian, Z. Mei, P. Bodong, Z. Jizhen, Y. Zhiqin, W. Fuli, and Y. Yuan, Optical diagnostics of multigap gas switches for linear transformer drivers, Plasma Sci. Technol. 16, 677 (2014).

[22] G. A. Mesyats, Generating powerful nanosecond pulses (Soviet Radio, USSR, Moscow, 1974).

[23] S. I. Braginskii, Theory of the development of a spark channel, Sov. Phys. JETP 34, 1068 (1958).

[24] S. I. Andreev and B.I. Orlov, On the theory of the development of the spark discharge, Sov. Phys. Tech. Phys. 30, 1097 (1966). 